\title{
Resistencia in vitro de la garrapata equina (Dermacentor nitens) en el departamento de Córdoba, Colombia
}

\author{
Resistance in vitro equine tick (Dermacentor nitens) in the Department of \\ Córdoba, Colombia
}

\author{
Montañez B, Yonatan ${ }^{1,2}$ MVZ, Gutiérrez P, Delio ${ }^{1,2}$ MVZ, Martínez H, Nicolas ${ }^{1}$ M.Sc, \\ Herrera B, Yonairo ${ }^{1 *}$ MVZ, Vergara G, Oscar ${ }^{1}$ Ph.D.
}

${ }^{1}$ Universidad de Córdoba. Facultad de Medicina Veterinaria y Zootecnia.
Departamento de Ciencias Pecuarias. Montería. Colombia.
${ }^{2}$ Grupo de Estudio en Parasitología y Salud Publica - REDIA.

\section{Key words:}

Acaricide; metrifonate; cypermethrin; amitraz.

\section{Abstract}

Determine the resistance of the tick Dermacentor nitens to metrifonate (Neguvon ${ }^{\circledR}$, Bayer $^{\odot}$, Germany), cypermethrin (Ganabaño ${ }^{\circledR}$, Novartis ${ }^{\odot}$, USA), and amitraz (Triatox ${ }^{\circledR}$, Schering Plough ${ }^{\odot}$, USA). A non-probabilistic convenience experimental study, five plots where teleogines 120 engorged $D$. nitens were collected, the teleogines were subjected to immersion test according to Drummond. It was found that the treatment was less chemoresistance produced amitraz $97 \%$ effective, followed by $93 \%$ cypermethrin and $53 \%$ respectively metrifonate. An oviposition evaluation, it was found that amitraz is the most effective $23 \%$, cypermethrin $18 \%$ and then metrifonate 1\%. The percentage hatching assessment was higher for metrifonate $18.97 \%$, cypermethrin $4.70 \%$ and Amitraz $1.68 \%$ respectively. It is concluded that amitraz is the most effective acaricide in vitro not only affect adults but to eliminate the cycle of $D$. nitens, but its use in practice is unlikely in vivo by toxic effects in horses. Among the products used in horses, cypermethrin is the drug of choice.

\section{Resumen}

Determinar la resistencia de la garrapata Dermacentor nitens a metrifonato (Neguvon $^{\circledR}$, Bayer $^{\odot}$, Alemania), cipermetrina (Ganabaño ${ }^{\circledR}$, Novartis $^{\odot}$, USA), y amitraz (Triatox ${ }^{\circledR}$, Schering Plough $^{\odot}$, USA). Un estudio experimental no probabilístico por conveniencia, de cinco predios, donde se recolectaron 120 teleogínas ingurgitadas de $D$. nitens, las teleogínas fueron sometidas a inmersión según prueba de Drummond. Se encontró que el tratamiento que menos quimioresistencia produjo fue amitraz con $97 \%$ de efectividad, seguido por cipermetrina con $93 \%$ y metrifonato con $53 \%$ respectivamente. A la evaluación de la oviposición, se encontró que amitraz es el de mayor efectividad $23 \%$, luego cipermetrina $18 \%$ y metrifonato con $1 \%$. La evaluación porcentual de eclosión fue mayor para el metrifonato con $18,97 \%$, cipermetrina con $4,70 \%$ y amitraz con $1,68 \%$ respectivamente. Se concluye que amitraz es el acaricida más efectivo in vitro no solo para eliminar adultos sino afectar el ciclo de $D$. nitens, pero su uso en la práctica in vivo es improbable por los efectos tóxicos en équidos. Entre los productos utilizables en los equinos, la cipermetrina es el fármaco de elección. 


\section{Introducción}

Los equinos, pueden infestarse por variedades de ectoparásitos, entre los cuales se destaca la garrapata, un parásito externo que infesta animales domésticos, silvestres y al hombre, su distribución es casi mundial (FELGUEROSO, 2011). Las garrapatas poseen importancia sanitaria y económica, debido a su acción hematófaga, vectorial de patógenos e inoculación de toxinas. Son consideradas como el segundo grupo de vectores (después de mosquitos) de importancia en la transmisión de enfermedades infecciosas a humanos y animales en el ámbito mundial (CORTES, 2011). Se han propuesto varios tipos de control dentro del manejo integrado de garrapatas, como control biológico, químico y físico (LINARES, 2010).

En Colombia, el control de este parásito se realiza casi exclusivamente con acaricidas químicos (GARCÍA, 2011). Sin embargo, la mayoría de la resistencia a los acaricidas es un fenómeno creciente en todo el mundo; Por lo tanto, es necesario identificar las estrategias para aumentar su vida útil y para disminuir la dependencia de los controles químicos (MONCADA et al., 2015).

El desarrollo de poblaciones de garrapatas resistentes a los acaricidas se da como resultado de la eliminación constante de individuos genéticamente susceptibles a cualquiera de estas sustancias, fomentando la supervivencia de los individuos insensibles (resistentes) (ALONSO-DÍAZ et al., 2006). Los mecanismos que confieren la resistencia varían entre individuos, y los mejor descritos, hasta el momento, incluyen la insensibilidad en el sitio de acción, la resistencia metabólica y la disminución en la permeabilidad de la cutícula de la garrapata (ALONSO-DÍAZ et al., 2006; GUERRERO et al., 2012).

La resistencia desarrollada por las garrapatas frente a los acaricidas de síntesis, hace necesario replantear las estrategias utilizadas actualmente para su control (MONCADA et al., 2015). En la actualidad no hay información reportada a cerca de la resistencia que presenta $D$. nitens a los ectoprasiticidas para su control en los equinos; debido a ello el objetivo principal del presente estudio fue determinar el grado de resistencia in vitro de la garrapata $D$. nitens a ectoparasiticidas en equinos del departamento de Córdoba, Colombia.

\section{Materiales y métodos}

Se realizó un estudio experimental. Para la investigación se visitaron cinco fincas de tres municipios ubicados en el departamento de Córdoba, Colombia, ubicado entre las coordenadas $7^{\circ} 23^{\prime}$ y $9^{\circ} 26^{\prime}$ de latitud norte y los $74^{\circ} 52^{\prime}$ y $76^{\circ} 32^{\prime}$ de longitud oeste, a una altura de
$30 \mathrm{msnm}$, con temperatura promedio anual de $28^{\circ} \mathrm{C}$, humedad relativa del $84 \%$, precipitación media anual de $1200 \mathrm{~mm}$ y pertenece a la formación climática de bosque tropical lluvioso (PABÓN et al., 2001; CASSAB et al., 2010).

Se emplearon 120 garrapatas adultas ingurgitadas, con un tamaño mayor a $5 \mathrm{~mm}$. Estas fueron distribuidas aleatoriamente en grupos de 30 individuos, fueron sometidas a tres tratamientos y un placebo, los productos fueron metrifonato, cipermetrina y amitraz. Se utilizó la técnica de inmersión de adultos descrita por DRUMMOND et al. (1973); en la cual cada grupo de garrapatas fue sumergido durante tres minutos en diluciones de los fármacos utilizados a la dosis recomendada por el fabricante, metrifonato $1.5 \mathrm{~g}$ por 1 litro de agua, cipermetrina $150 \mathrm{mg} / \mathrm{ml}$ : $1 \mathrm{ml}$ por litro de agua y amitraz $125 \mathrm{mg} / \mathrm{ml}: 1 \mathrm{ml}$ por litro de agua. Para cada tratamiento se realizaron tres repeticiones o replicas (BENAVIDES et al., 2008). Posterior a cada tratamiento, se adhirieron las garrapatas por la parte dorsal de su cuerpo a cintas adhesivas dentro de cajas Petri y se ubicaron en diferentes direcciones craneal - caudal. Se Incubaron a temperatura promedio de $27^{\circ} \mathrm{C}$ y una humedad relativa de $85 \%$ durante 18 días. Iniciada la incubación (Incubadora BD-115, RS422), diariamente se contaron las garrapatas que morían, las que sobrevivían, las que oviposicionaron y aquellas que aunque llegaron a depositar huevos se inhibió o no la eclosión de los respectivos huevos. La masa total de huevos producidos por cada garrapata al final del periodo de ovoposición (ootoquia) a los 18 días pos tratamiento, se colectaron en tubos de ensayo, tapándolos con algodón e incubándolos durante 21 días bajo las mismas condiciones de humedad y temperatura señaladas anteriormente y así determinar el porcentaje de eclosión (BRAVO et al., 2008).

Los resultados obtenidos fueron tabulados en Microsoft Excel $^{\circledR} 2010$ y analizados mediante estadística descriptiva para las variables supervivencia, ovoposición y eclosión. Además, para el análisis de supervivencia se utilizó la prueba de Kaplan - Meier y se determinó mediante la prueba de Chi Cuadrado si el tratamiento aplicado era independiente de la oviposición y de la supervivencia. Los análisis se realizaron en el programa estadístico InfoStat 2015 e.

\section{Resultados}

En la Tabla 1 se observa que de 120 teleoginas totales de $D$. nitens sometidas a diferentes tratamientos, sólo sobrevivieron $17(14,16 \%)$, de las cuales $14(47 \%)$ correspondieron al tratamiento con metrifonato $2(7 \%)$ a cipermetrina y $1(3 \%)$ para amitraz respectivamente; en base a los 30 individuos por cada tratamiento. Se pudo encontrar que el $81 \%$ de las garrapatas son susceptibles al tratamiento mientras se encontró $19 \%$ de resistencia en el global. 
Tabla 1. Tabla de frecuencias para la supervivencia de $D$. nitens.

\begin{tabular}{cllll}
\hline Tratamiento & \multicolumn{2}{c}{ Mortalidad } & \multicolumn{2}{c}{ Supervivencia } \\
\hline & $\mathrm{N}$ & $\%$ & $\mathrm{~N}$ & $\%$ \\
amitraz & 29 & 97 & 1 & 3 \\
cipermetrina & 28 & 93 & 2 & 7 \\
metrifonato & 16 & 53 & 14 & 47 \\
\hline Total & $\mathbf{7 3}$ & $\mathbf{8 1}$ & $\mathbf{1 7}$ & $\mathbf{1 9}$ \\
\hline
\end{tabular}

En Figura 1, se muestra el porcentaje de supervivencia durante el periodo de incubación de teleogínas. Se encontró diferencia altamente significativa $(p=0,000152)$ para el porcentaje de supervivencia entre los grupos experimentales. Además, se observa que la distribución que toma la supervivencia de las $D$. nitens evaluadas en en el periodo experimental (18 días), con relación a los tratamientos a las que fueron sometidas, se aprecia que a través del tiempo la supervivencia de las garrapatas se ve afectada por los tratamiento aplicados. El grupo control, aunque solo fue sumergido en agua destilada, presento una reducción en la supervivencia, esto se puede atribuir a que posiblemente los productores pudieron haber aplicado algún tratamiento anterior a la colecta de las garrapatas; aunque a la hora de encuestarlos aseguraron no haber realizado control ectoparaticida en los últimos treinta días.
En la Tabla 2 se observa que de 120 teleoginas totales de $D$. nitens adultas sometidas a los diferentes tratamientos, 75 (62,5\%) ovoposicionaron; de estas 29 $(96,6 \%)$ correspondieron al tratamiento con metrifonato, $27(90 \%)$ al control, $12(40 \%)$ a cipermetrina y 7 $(23,33 \%)$ para amitraz respectivamente. Del total de garrapatas expuestas a la inmersión de los diferentes grupos acaricidas que ovoposicionaron, el $62,5 \%$ ovoposicionó, mientras que el $37,5 \%$ no ovoposicionó.

Tabla 2. Valores de frecuencias de ovoposición absoluta y relativa, para $D$ nitens.

\begin{tabular}{ccccc}
\hline Tratamiento & \multicolumn{2}{c}{$\begin{array}{c}\text { Garrapatas que no } \\
\text { oviposicionaron }\end{array}$} & \multicolumn{2}{c}{$\begin{array}{c}\text { Garrapatas que } \\
\text { oviposicionaron }\end{array}$} \\
\hline amitraz & $\mathrm{N}$ & $\%$ & $\mathrm{~N}$ & $\%$ \\
cipermetrina & 23 & 76,67 & 7 & 23,33 \\
metrifonato & 18 & 60 & 12 & 40 \\
control & 1 & 3,3 & 29 & 96,6 \\
\hline Total & 3 & 10 & 27 & 90 \\
\hline
\end{tabular}

En la Tabla 3, se observa que el tratamiento que obtuvo el mayor porcentaje de eclosión fue el metrifonato con un $18,97 \%$, seguido por el control con $16.02 \%$, cipermetrina con $4,70 \%$ y por último el amitraz con $1,68 \%$.

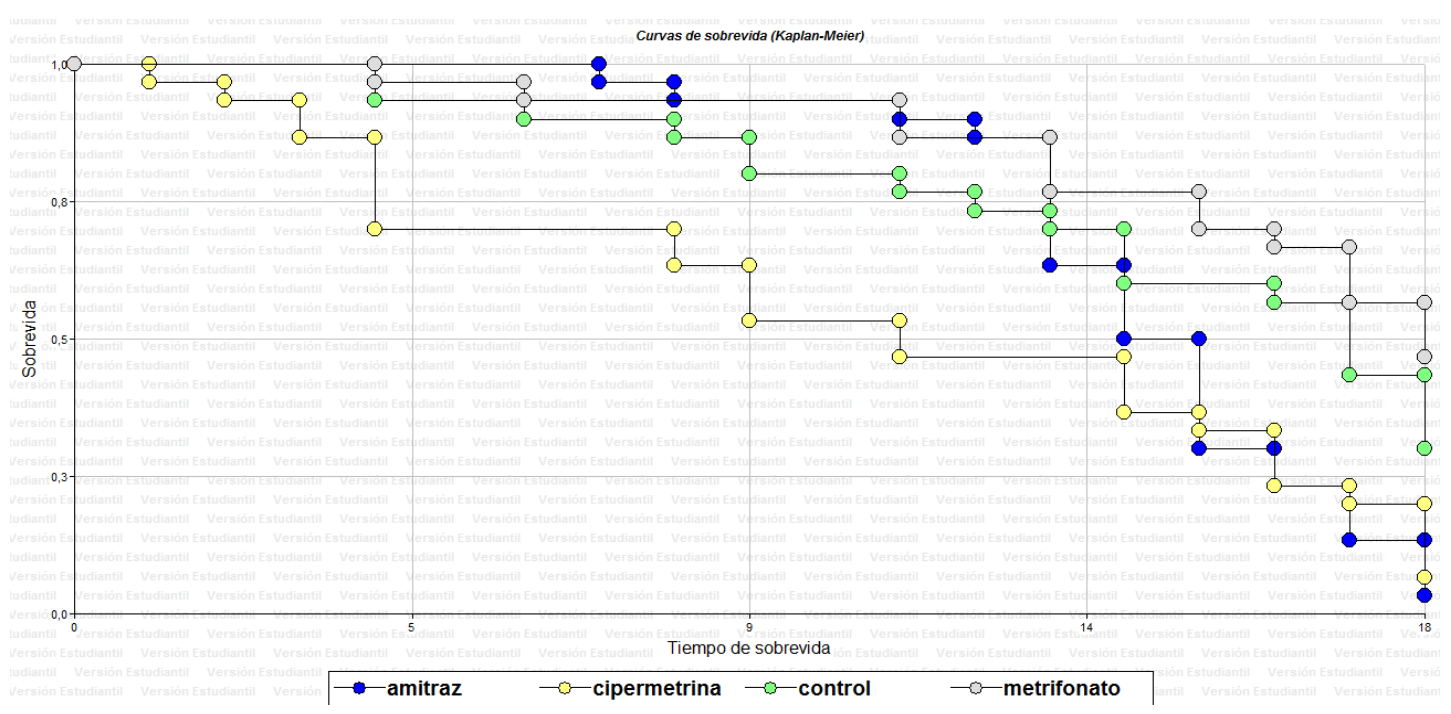

Figura 1. Grafica de supervivencia poblacional de garrapata $D$. nitens según prueba de Kaplan-Meier

$\begin{array}{ccccc}\text { Tratamientos } & \text { Total de huevos } & \text { Porcentaje de huevos } & \text { Total de ninfas } & \text { Porcentaje de ninfas } \\ \text { control } & 18703 & 83,97 \% & 3569 & 16,02 \% \\ \text { amitraz } & 2456 & 98,31 \% & 42 & 1,68 \% \\ \text { cipermetrina } & 9342 & 95,29 \% & 461 & 4,70 \% \\ \text { metrifonato } & 20204 & 81,02 \% & 4730 & 18,97 \%\end{array}$

Tabla 3. Porcentaje de eclosión de $D$. nitens sometido a inmersión 
Fue realizada prueba de $\mathrm{Chi}^{2}$ en la cual se encontró dependencia entre los tratamientos aplicados con la ovoposición $(p \leq 0,01)$, la dependencia también se presenté entre tratamientos y supervivencia $(p \leq 0,01)$. Lo cual indica que los tratamientos tiene un efecto directo sobre las variables supervivencia, ovoposición y eclosión de larvas, en el presente estudio in vitro.

\section{Discusión}

De los tratamientos utilizados, el metrifonato fue el menos efectivo para controlar el ciclo reproductivo de la garrapata del equino ( $47 \%$ de supervivencia y $96,6 \%$ de ovoposición). Sin embargo, los demás compuestos, amitraz y cipermetrina, resultaron ser eficaces contra las teleoginas, mostrando la baja resistencia que esta posee frente a estos principios activos, lo cual no coincide con la información descrita para $R$. microplus por RUIZ y BLANCO (2009), en el Pie de Monte Llanero colombiano, en el cual los tratamientos con cipermetrina y amitraz arrojaron resultados de quimioresistencia del $56,8 \%$ y $56,5 \%$ respectivamente. Esto podría deberse a la limitación y restricción que tiene el amitraz en equinos.

Recién se publicó un estudio donde se evaluó la resistencia in vitro de $R$. microplus a acaricidas en dos fincas el norte del Tolima, el estudio revelo una eficacia del $51,95 \%$ y $76,93 \%$ en cada finca para el amitraz, seguido de la cipermetrina con un $87,85 \%$ y $93,5 \%$, no obstante el ethion mostro un nivel de efectividad del 100\% (DIAZ, 2015). Estos datos, muestran que el amitraz por su uso frecuente en bovinos ha creado quimioresistencia por parte de la garrapata hacia este principio activo.

Los resultados de esta investigación, no coinciden con el estudio realizado por ESCOBAR (1995), el cual determinó que en Colombia se han utilizado productos pertenecientes a todos los grupos químicos disponibles para el control de la garrapata como diclorvos, bendiocarba, propoxur, triclorform, entre otros; generando resistencia en las poblaciones de estas, a diferencia de lo encontrado en la presente investigación, los ixodidos no mostraron resistencia a las moléculas cipermetrina y amitraz (RUIZ y BLANCO, 2009).

Estudios revelan lo contrario a resultados encontrados en este experimento, pero en la especie bovina, donde REYES et al. (2013) comprobaron en el municipio de San Juan Evangelista, Veracruz, México, la existencia de cepas de garrapatas $R$. microplus con resistencia múltiple a amitraz propiciado por las malas prácticas de manejo durante el baño garrapaticida, que a su vez derivan de falta de asesoría profesional para su apropiada realización, caso contrario ocurrido en esta investigación donde $D$. nitens resultó ser altamente sensible a esta molécula. Esto debido a que la garrapata no ha sido expuesta a este principio farmacológico y por ende no ha creado la capacidad de resistencia a diferencia, de las explotaciones bovinas donde su uso es muy frecuente.

Investigaciones realizadas por BENAVIDES et al. (1989) y BETANCOURT (1993) reportaron poblaciones de garrapatas resistentes a moléculas como flumetrina, deltametrina, a cipermetrina, lamdacialotrina y cipermetrina, situación que no coincide con lo encontrado en las poblaciones de garrapatas $D$. nitens estudiadas en el departamento de Córdoba, cuya quimiorresistencia a la cipermetrina es considerablemente baja (RUIZ y BLANCO, 2009).

Según ANDREW et al. (2003) la garrapata R. microplus ha desarrollado en la mayoría de los países un grado importante de resistencia a organofosforados, la cual es causada por mecanismos de detoxificación y por alteraciones o insensibilidad de la acetilcolinesterasa, que es su sitio o blanco de acción en insectos y arácnidos. En las poblaciones estudiadas de $D$. nitens de este experimento se evidenció una alta resistencia al metrifonato, lo que muestra que el problema de quimioresistencia a los ixodicidas también afecta a la garrapata de la especie equina, la cual se está viendo influenciada por el manejo irracional de las infestaciones de estas. La resistencia a acaricidas también tiene un impacto importante sobre la incidencia de las enfermedades causadas por los hemoparásitos transmitidos por garrapatas, debido a que aumenta el número de éstas por animal y por ende las posibilidades de que se incremente la transmisión de estos padecimientos, además de aumentar los costos de producción por el tratamiento de la patología causada por el ente infeccioso transmitido.

El problema de la resistencia a los acaricidas químicos es un fenómeno a nivel mundial que cada día se hace más visible y afecta particularmente las zonas tropicales y subtropicales, siendo uno de los agravantes principales la dependencia casi exclusiva, para el control parasitario, de las sustancias químicas. Aunado a lo anterior, como lo indica la FAO, se encuentra la falta de investigación para la obtención de nuevas moléculas eficaces, debido al alto costo que ello representa. (ALVAREZ y HERNANDEZ, 2010).

Existen elementos que tienen relevancia a la hora de analizar la sistematicidad para realizar el control de la infestación por garrapatas como son: la utilización de vehículos para la aplicación de principios activos, el uso de aguas para la dilución del producto, la asistencia técnica, la dosis a aplicar de los productos, cantidad de la solución a asperjar, según peso animal, etc. Todo lo anterior, afecta de forma directa el resultado final, que es el control de las poblaciones de garrapatas sobre 
el animal, y por las respuestas obtenidas se observa la presencia de tales fallas de manejo, reflejado en los niveles de infestaciones que los animales poseían y en la desinformación por parte de los productores.

\section{Conclusión}

Se comprobó que la garrapata $D$. nitens posee resistencia en un alto porcentaje al organofosforado metrifonato. El amitraz (Amidinas) fue el compuesto que menos resistencia presentó, con un $3 \%$ de supervivencia por parte de $D$. nitens. A pesar de la alta efectividad del amitraz frente a la garrapata del equino, no es recomendable la aplicación de productos comerciales a base de este compuesto por los efectos toxicológicos que ocasiona sobre esta especie. La ovoposición de la garrapata depende en gran medida del principio activo a la cual es sometida. Se recomienda el uso de la cipermetrina como un producto eficaz en el control de este ectoparásito. A nivel nacional esta investigación es el primer reporte realizado sobre la resistencia de la garrapata del equino a diferentes productos ixodicidas.

\section{Referencias}

ALONSO-DÍAZ, M.A.; RODRÍGUEZ-VIVAS, R.I.; FRAGOSO-SÁNCHEZ, H. y ROSARIO-CRUZ, R. 2006. Resistencia de la garrapata Boophilus microplus a los ixodicidas. Arch Med Vet. 38 (2):105-13.

ÁLVAREZ, V.; HERNÁNDEZ, V. 2010. Diagnóstico de resistencia a organofosforados, piretroides sintéticos, amidinas e ivermectinas en la garrapata Rhipicephalus microplus en fincas de productores de leche de Costa Rica. Rev FAVE $9(2): 7-17$

ANDREW, L.; DAVEY, R.; MILLER, R.; GEORGE, J. 2003. Resistance to Coumaphos and Diazinon in Boophilus microplus (Acari: Ixodidae) and evidence for the involvement of an oxidative detoxification mechanism. $\mathrm{J}$ of Med Entomol. 40:482- 490.

BENAVIDES, O.; ROMERO, E.; RODRÍGUEZ, B. 1989. Situación actual de la resistencia de la garrapata Boophilus microplus a acaricidas en Colombia. El diagnóstico de resistencia. Carta Fedegan 61:13-18.

BETANCOURT, J. Situación actual de las garrapatas en Colombia. I Foro nacional sobre la situación de las garrapatas y moscas en la ganadería. Bogotá, 1993. 10-15.

BRAVO, M.J.; CORONADO, A.; HENRÍQUEZ, H. 2008. Susceptibilidad de larvas y adultos de Boophilus microplus al ixodicida coumafos en explotaciones lecheras del estado Lara, Venezuela 26 (1):41-46.

CASSAB, A.; MORALES, V.; MATTAR, S. 2010. Factores climáticos y casos de Dengue en Montería, Colombia. 2003-2008. Rev. Salud pública 13 (1):115-128.

CORTES, J. 2011. Garrapatas: estado actual y perspectivas. Biomédica 31 (3):3-315.

DIAZ, E. 2015. Evaluación in vitro de resistencia a acaricidas en Rhipicephalus microplus. Estudio de caso en el norte del Tolima, Colombia. RCCA 8 (1):37-43.

DRUMMOND, R.; ERNST, S.; TREVINO, J.; GLADNEY, W.; GRAHAM, O. 1973. Boophilus annulatus and Boophilus microplus: Laboratory test of insecticides. J. Econ. Entomol. 66:130-133.

ESCOBAR, A.1995. Control a base de productos químicos. Foro regional del magdalena medio sobre "La situación de las garrapatas y moscas en la ganadería" Asociación Nacional de Laboratorios de Productos Veterinarios. Puerto Salgar, Cundinamarca.

FELGUEROSO, A. 2011. Las garrapatas como agentes transmisores de enfermedades para los animales y el hombre. Serida. Asturias.

GARCÍA PAZ JL. 2011. Evaluación de las propiedades acaricidas de Piper crassinervium Kunth. Piper aequale Vahl. (Piperaceae) sobre larvas de Rhipicephalus (Boophilus) microplus. Trabajo de grado para optar a título de maestría. Universidad Nacional. Colombia. 
GUERRERO, F.D.; LOVIS, L. y MARTINS, J.R. 2012. Acaricide resistance mechanisms in Rhipicephalus (Boophilus) microplus. Brazilian J Vet Parasitol. 21 (1):-6.

LINARES, V. Manejo integral de las garrapatas una propuesta eficiente y sostenible con el medio ambiente. Manizales, Colombia; 2010.

MONCADA, G.; VILLAR, D.; CHAPARRO, G.; ANGULO, A. y MAHECHA, L. 2015. Aproximación al uso de hongos entomopatógenos y vacunas para el control sostenible de garrapatas en sistemas ganaderos: revisión. AIA 19 (3):55-71.

PABÓN, J.; ESLAVA, J.; GÓMEZ, R. 2001. Generalidades de la distribución espacial y temporal de la temperatura del aire y de la precipitación en Colombia. Meteorol Colomb. 4:47-59

REYES, D.; ARIETA, R.; FERNÁNDEZ, F.; ROMERO, F.; PENICHE, C. 2013. Resistencia de Rhipicephalus (boophilus) microplus a ixodicidas en ranchos bovinos del municipio de San Juan Evangelista, Veracruz, México. Redvet. 14 (7). Disponible en: http://www.veterinaria.org/revistas/redvet/n070713/071304.pdf. Consultada: 10-01-2015.

RUIZ, M.; BLANCO, N. 2009. Grado de resistencia del Rhipicephalus (Boophilus) microplus a productos ixodicidas, y su residualidad en leche en 20 predios del sistema doble propósito del Piedemonte Llanero. Tesis de grado. Universidad de la Salle, Bogotá, Colombia. 